\title{
Efeitos do estímulo verbal sobre o desempenho no teste de escada e ajustes cardiorrespiratórios em sujeitos saudáveis
}

\author{
The effects of verbal stimulus on performance in stair climbing test and \\ cardiorespiratory adjustments in healthy subjects
}

Heloisa Borges', Doralice Fernanda da Silva Raquel', Priscila Machado Batista', Robison José Quitério²,3, Alexandre Ricardo Pepe Ambrozin²

RESUMO I Esta pesquisa teve o objetivo de avaliar os efeitos do estímulo verbal (EV) no tempo do teste de escada (TESC) e nas variáveis cardiorrespiratórias de adultos saudáveis. Trinta e um adultos saudáveis realizaram dois TEsc (com EV e sem EV). Antes e depois de cada teste, foram avaliados os sinais vitais e a Escala de Borg. Os tempos nos TEsc foram comparados por meio do Teste $t$ de Student para amostras pareadas e as diferenças, de acordo com a ordem de realização dos testes utilizando o Teste de MannWhitney. Os sinais vitais e a Escala de Borg foram comparados por meio do Teste de Friedman ou ANOVA com post hoc do Teste de Tukey. As variações foram comparadas utilizando o Teste $t$ Student para amostras independentes ou Teste de Mann-Whitney ( $p<0,05)$. O tempo no TEsc sem EV foi de 23,48 $\pm 8,28$ segundos, significativamente maior que o teste com EV, que foi de 21,60 $\pm 7,18$ segundos ( $p<0,05$ ). Todas as variáveis aumentaram após os testes, e a Escala de Borg foi a única que teve maior variação no TEsc com

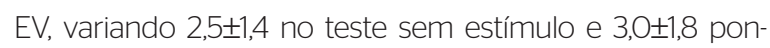
tos no com estímulo $(p<0,05)$. O estímulo verbal melhora o desempenho no TEsc e leva à maior sensação de esforço. Descritores I teste de esforço; motivação; distribuição aleatória; testes de função respiratória.
ABSTRACT I This research aimed to evaluate the effects of verbal stimuli (VS) in the time of the Stair climbing Test (SCT) and in the cardiorespiratory variables on healthy adults. Thirty-one healthy adults performed two SCT (with VS and without VS). Before and after each test, vital signs and Borg Scale were evaluated. The times in SCT were compared using the Student's t-test for paired samples, and differences were compared according to the order of the testing using the Mann-Whitney Test. The vital signs and Borg Scale were compared by the Friedman Test or ANOVA with post-hoc Tukey Test. The variations of these variables were compared using the Student's $t$-test for independent samples or Mann-Whitney Test ( $p<0.05)$. The time in the SCT without VS was $23.48 \pm 8.28$ seconds significantly greater than the test with VS that was $21.60 \pm 7.18$ seconds $(p<0.05)$. All the variables increased after the tests and the Borg Scale was the one that had more variation in SCT with VS, ranging $2.5 \pm 1.4$ in the test without VS and $3.0 \pm 1.8$ points in the test with VS $(p<0.05)$. Verbal stimulation improves performance in TEsc and leads to greater sensation of effort.

Keywords I exercise test; motivation; random allocation; respiratory function tests.

Estudo desenvolvido na Faculdade de Filosofia e Ciências, Centro de Estudos de Educação para Saúde, Universidade Estadual Paulista "Júlio de Mesquita Filho" (UNESP) - Marília (SP), Brasil.

'Discentes do curso de Fisioterapia, Departamento de Fisioterapia e Terapia Ocupacional, UNESP - Marília (SP), Brasil. ${ }^{2}$ Professor Assistente Doutor do Departamento de Fisioterapia e Terapia Ocupacional da UNESP - Marília (SP), Brasil.

3Docente do Programa de Pós-graduação em Desenvolvimento Humano e Tecnologias, Instituto de Biociências, UNESP - Rio Claro (SP), Brasil. 


\section{INTRODUÇÃO}

O teste de escada (TEsc) há muito tempo vem sendo utilizado na avaliação da condição cardiopulmonar ${ }^{1}$; porém, diferente do teste de caminhada de seis minutos (TC6) $)^{2}$ o TEsc ainda não foi protocolado, especialmente quanto ao uso do estímulo verbal (EV). Sabe-se, por exemplo, que o EV pode aumentar significativamente a distância percorrida no TC6 $6^{3,4}$.

As pesquisas que utilizam o TEsc em pacientes cirúrgicos não limitam a altura da escada, não consideram o tempo de subida e poucas referem a utilização de $\mathrm{EV}^{1,5,6}$. Cataneo et al. ${ }^{6}$ fixou a altura em 12 metros e passou a utilizar como variável o tempo de subida, com EV constante.

Alguns autores acreditam que o EV deve ocorrer durante todo o teste ${ }^{5,7}$, porém não encontramos trabalhos que comparem TEsc realizados com e sem EV.Em pesquisa anterior, o EV reduziu o tempo no TEsc de sujeitos saudáveis, mas a ordem dos testes não foi randomizada ${ }^{7}$. Realizar TEsc com sujeitos saudáveis permite conhecer as respostas fisiológicas normais frente diante do teste já que pacientes pneumopatas ou cardiopatas podem apresentar limitações físicas decorrentes da doença que limitam o desempenho no teste.

Além disso, saber os efeitos do TEsc nas variáveis cardiorrespiratórias é fundamental, já que esse teste busca reproduzir o estresse cardiorrespiratório gerado pelo procedimento cirúrgico ${ }^{8}$ e que a dessaturação já mostrou-se capaz de predizer o risco de complicação pós-operatória ${ }^{9}$.

OTEsc pode auxiliar na prescrição de exercícios. Para tanto, é necessário conhecer seus efeitos em sujeitos saudáveis e, posteriormente, padronizar em portadores de doenças, especialmente quanto à utilização do EV. Assim, o objetivo desta pesquisa foi avaliar quais são os efeitos do estímulo verbal no tempo do TEsc quando os testes são aplicados em ordem aleatória e avaliar os efeitos do teste nas variáveis cardiorrespiratórias de adultos saudáveis.

\section{METODOLOGIA}

Após aprovação do Comitê de Ética e Pesquisa da Instituição (Parecer $n^{\circ}$ 0152/2011), foram convidados 42 indivíduos saudáveis maiores de 18 anos que trabalhavam e/ou frequentavam a Instituição. Seis se recusaram a participar, quatro não atingiram os critérios de inclusão e um não finalizou o protocolo.

Assim, foram incluídos 31 indivíduos de ambos os sexos com idade 50,32 $\pm 17,10$ anos, que negaram doenças cardiorrespiratórias, como angina instável, infarto do miocárdio a menos de três meses, bradicardia, taquicardia, hipertensão arterial sistêmica descontrolada, insuficiência cardíaca ou doença pulmonar obstrutiva crônica descompensada. Os indivíduos não apresentavam alterações neurológicas, vasculares, ortopédicas ou músculo-esqueléticas que impedissem a realização dos testes ou frequência cardíaca (FC) em repouso acima de 120 bpm.

Foram registrados idade, sexo e presença de comorbidades, e todos responderam um questionário para avaliação do nível de atividade física ${ }^{10}$. O Índice de Massa Corporal (IMC) foi obtido dividindo-se a massa $(\mathrm{kg})$ pela estatura ao quadrado $\left(\mathrm{m}^{2}\right)^{11}$. A massa foi verificada em balança digital (FILIZOLA ${ }^{\circledR}$ ), e a estatura foi obtida em estadiômetro graduado em centímetros $(\mathrm{cm})$.

A espirometria foi realizada segundo os critérios da American Thoracic Society ${ }^{12}$ em espirômetro One Flow Clement Clarke International. Foram obtidas três provas de capacidade vital forçada (CVF), sendo a CVF e volume expirado forçado no primeiro segundo $\left(\mathrm{VEF}_{1}\right)$ apresentados em litros, e a relação $\mathrm{VEF}_{1} / \mathrm{CVF}$ em porcentagem.

Posteriormente, os indivíduos realizaram dois TEsc (com e sem EV), respeitando o intervalo de uma hora entre eles. A ordem de realização dos testes foi aleatorizada por meio de sorteio (G1 - TEsc sem EV primeiro - 17 indivíduos; G2 - TEsc com EV primeiro - 14 indivíduos).

Antes de cada teste, os indivíduos foram colocados em repouso e registrados os sinais vitais e a sensação de esforço. Os valores de pressão arterial sistólica (PAS) e diastólica (PAD) foram verificados utilizando esfigmomanômetro (Premium Manual-ESFHS501; Glicomed) e estetoscópio (Rappaportt; Glicomed) ${ }^{13}$. A frequência respiratória (FR), a FC e a saturação de oxigênio $\left(\mathrm{SpO}_{2}\right)$ também foram registradas, sendo a primeira por meio da observação da expansão da caixa torácica e as demais verificadas por um oxímetro de pulso (modelo 9500 Onyx) posicionado no segundo dedo da mão esquerda. A porcentagem da FC máxima (\%FCmáx) foi obtida a partir da fórmula: \%FCmáx=(FCregistradax100)/(202-idade). A sensação de dispneia foi pontuada pelos participantes de acordo com a Escala de Borg modificada ${ }^{14}$.

Os TEsc foram realizados em uma escada à sombra, composta por quatro lances, 46 degraus de $16 \mathrm{~cm}$ de altura cada um, totalizando 7,36 $\mathrm{m}$ de altura, com inclinação de $30^{\circ}$. Os indivíduos foram orientados a subir o mais rápido possível, não sendo permitido correr, e o tempo de subida foi registrado em segundos utilizando cronômetro acionado a partir do momento em que o individuo colocasse o pé no primeiro degrau e parado quando este colocasse os dois pés no último degrau. 
O TEsc com EV foi realizado com acompanhamento do pesquisador, que estimulava o indivíduo com frases padronizadas a cada lance da escada ("Vai mais rápido!"; "Você está indo bem!" e "Falta pouco!"). O estímulo foi dado sempre pelo mesmo avaliador, no mesmo tom, não empregando linguagem corporal como forma de incentivo. No TEsc sem EV, o pesquisador somente acompanhava o indivíduo sem estimulá-lo. No final do teste, as variáveis cardiorrespiratórias e a sensação de esforço foram verificadas novamente.

Os testes seriam interrompidos caso o indivíduo relatasse fadiga, dispneia intensa, dor torácica, exaustão ou julgassem necessário. Os indivíduos que apresentassem PAS maior ou igual a $200 \mathrm{mmHg}$, PAD maior ou igual a $100 \mathrm{mmHg}, \mathrm{FC}$ menor que $40 \mathrm{bpm}$ ou maior que $120 \mathrm{bpm}, \mathrm{SpO}_{2}$ abaixo de $90 \%$ ou FR maior que $25 \mathrm{ipm}$ antes da realização dos testes seriam excluídos da pesquisa.

Os dados foram submetidos ao Teste de normalidade de Kolmogorov-Smirnov, e os resultados apresentados em médiatdesvio padrão. O tempo no TEsc com e sem EV foram comparados utilizando o Teste $t$ de Student para amostras pareadas. Foram também comparadas as diferenças entre o teste com e sem EV no G1 e G2 utilizando o Teste de Mann-Whitney ( $\mathrm{p}<0,05)$.

Os sinais vitais e a Escala de Borg foram comparados nos diferentes momentos (antes e após TEsc com e sem EV) utilizando o Teste de Friedman ou ANOVA com post hoc do Teste de Tukey. As variações nos valores dos sinais vitais e Escala de Borg foram comparadas utilizando o Teste $t$ de Student para amostras independentes e o Teste de Mann-Whitney ( $\mathrm{p}<0,05)$.

\section{RESULTADOS}

As características dos 31 indivíduos (13 homens e 18 mulheres) são apresentadas na Tabela 1. Dos pacientes avaliados, oito indivíduos relataram hipertensão controlada e três eram ex-tabagistas com tempo de abs-

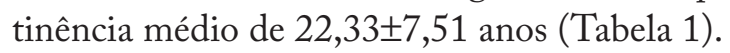

Quando comparados os tempos no TEsc, observou-se que no TEsc sem EV o tempo foi significativamente maior que no TEsc com EV, nos grupos (G1 e G2) e na amostra total (Figura). A diminuição entre os testes dos grupos foi de 1,18 segundos no $\mathrm{G} 1$ e de 1,52 segundos no $\mathrm{G} 2$ ( $\mathrm{p}=0,47$ ) (Figura).

Dentre as variáveis estudadas, todas aumentaram após os testes, independentemente do $\mathrm{EV}$, exceto a $\mathrm{PAD}$ e $\mathrm{SpO}_{2}$. Quando comparados os efeitos dos testes, por meio da variação antes e após o TEsc, a Escala de Borg foi a única que apresentou maior variação no TEsc com EV (Tabela 2).

Tabela 1. Dados antropométricos, nível de atividade física e função pulmonar de adultos saudáveis submetidos ao teste da escada sem e com estímulo verbal

$\begin{array}{lccc} & \text { Grupo } 1 & \text { Grupo } 2 & \text { Total } \\ \text { Homem/mulher (n) } & 8 / 9 & 5 / 9 & 13 / 18 \\ \text { Idade (anos)s } & 44,65 \pm 14,12 & 57,21 \pm 18,34 & 50,32 \pm 17,10 \\ \text { Massa (kg) } & 71,87 \pm 9,08 & 69,69 \pm 16,19 & 70,88 \pm 12,60 \\ \text { Estatura (m) } & 1,66 \pm 0,09 & 1,64 \pm 0,11 & 1,65 \pm 0,10 \\ \text { IMC (kg/m²) } & 26,09 \pm 2,36 & 26,01 \pm 4,99 & 26,05 \pm 3,71 \\ \text { Atividade física (pontos) } & 7,68 \pm 0,80 & 7,88 \pm 0,97 & 7,77 \pm 0,87 \\ \text { CVF (L) } & 4,02 \pm 0,77 & 3,51 \pm 1,08 & 3,81 \pm 0,93 \\ \text { VEF }(\mathrm{L}) & 3,25 \pm 0,77 & 2,77 \pm 0,85 & 3,05 \pm 0,82 \\ \text { VEF } / \text { CVF (\%) }_{\text {I }} & 79,00 \pm 57,89 & 78,00 \pm 0,10 & 79,66 \pm 7,56\end{array}$

IMC: índice de massa corporal; CVF: capacidade vital forçada; $\mathrm{VEF}_{\text {; }}$ volume expiratório forçado no primeiro segundo; L: litros; Idade e IMC (Teste de Mann-Whitney); Outras variáveis (Teste $t$ de Student para amostras independentes); ${ }^{\S}<<0,05$

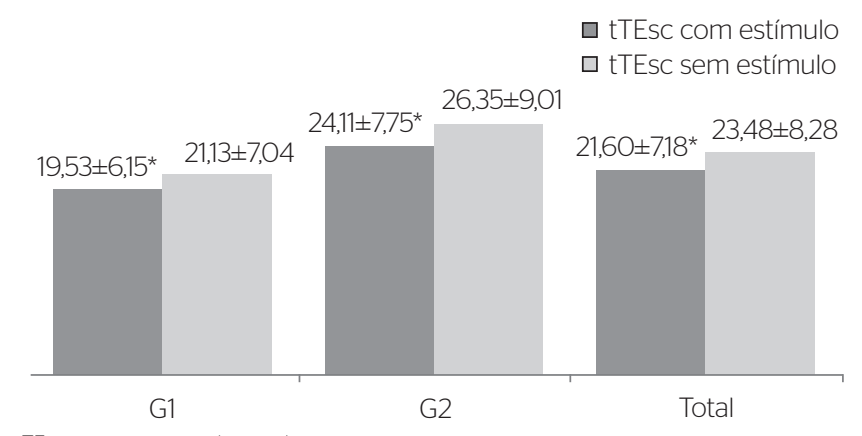

tTEsc: tempo no teste de escada

Figura. Valores do tempo no teste de escada sem e com estímulo verbal em segundos nos grupo 1 (G1) (sem estímulo primeiro), grupo 2 (G2) (com estímulo primeiro) e total; ( $\left.{ }^{*} \mathrm{p}<\mathrm{O}, \mathrm{O} 1\right)$;

Tabela 2. Média e desvio padrão das variáveis estudadas antes e após a realização dos testes de escada

\begin{tabular}{|c|c|c|c|c|c|c|}
\hline & \multicolumn{3}{|c|}{ Sem estímulo } & \multicolumn{3}{|c|}{ Com estímulo } \\
\hline & Antes & Após & $r$ & Antes & Após & $r$ \\
\hline PAS & $118,0 \pm 12,7$ & $146,7 \pm 23,5^{*}$ & $28,7 \pm 17,5$ & $118,1 \pm 12,4$ & $148,7 \pm 22,1^{*}$ & $30,6 \pm 19,2$ \\
\hline PAD & $73,5 \pm 8,3$ & $77,3 \pm 10,2$ & $7,3 \pm 3,7$ & $76,5 \pm 8,9$ & $70,7 \pm 22,0$ & $-5,7 \pm 23,9$ \\
\hline FR & $17,1 \pm 2,8$ & $22,6 \pm 3,4^{*}$ & $5,5 \pm 2,4$ & $17,8 \pm 2,8$ & $23,5 \pm 3,9^{*}$ & $5,7 \pm 3,1$ \\
\hline $\mathrm{SpO}_{2}$ & $96,9 \pm 1,4$ & $96,8 \pm 1,7$ & $-0,1 \pm 2,0$ & $97,2 \pm 1,3$ & $96,5 \pm 2,0$ & $-0,6 \pm 2,4$ \\
\hline FC & $80,0 \pm 11,5$ & $133,7 \pm 17,9^{*}$ & $53,7 \pm 15,9$ & $81,5 \pm 13,3$ & $136,4 \pm 17,8^{*}$ & $54,8 \pm 15,1$ \\
\hline \%FCmáx & $47,53 \pm 7,84$ & $79,01 \pm 9,22^{*}$ & $31,49 \pm 7,96$ & $48,40 \pm 8,61$ & $80,62 \pm 9,80^{*}$ & $32,22 \pm 7,91$ \\
\hline $\mathrm{EB}$ & $0,2 \pm 0,4$ & $2,6 \pm 1,5^{*}$ & $2,5 \pm 1,4$ & $0,1 \pm 0,3$ & $3,0 \pm 1,8^{*}$ & $2,9 \pm 1,7^{*}$ \\
\hline
\end{tabular}

*p<0.05; $\mathrm{SpO}_{2}$ : saturação de oxigênio (\%); FC: frequência cardíaca (bpm); \%FCmáx: porcentagem da frequência cardíaca máxima prevista; PAS: pressão arterial sistólica (mmHg); PAD: pressão arterial diastólica ( $\mathrm{mmHg}$ ); FR: frequência respiratória (ipm); EB: Escala de Borg; r: variação 


\section{DISCUSSÃO}

$\mathrm{O}$ estudo demonstrou que em adultos saudáveis o $\mathrm{EV}$ reduz o tempo do TEsc independentemente da ordem de aplicação dos testes. As características da amostra, quando comparada de acordo com a ordem de aplicação dos testes, foi homogênea, exceto na idade e nos tempos dos TEsc.

Em trabalho semelhante, a diminuição no tempo de TEsc foi de $28,9 \%$ com $\mathrm{EV}^{7}$, enquanto no presente estudo foi de 8,0\% na amostra total, 7,6\% no G1 e 8,5\% no G2. Embora as pesquisas mostrem que o $\mathrm{EV}$ melhorou o rendimento no TEsc, os diferentes resultados são decorrentes da não randomização da aplicação dos testes.

Sabe-se que em diversos testes de esforço o efeito aprendizado pode melhorar o rendimento do indivíduo ${ }^{2,15,16}$, daí a importância da randomização. Apesar de o tempo no TEsc com EV ser menor, não se pode descartar o efeito aprendizado, sendo interessante novos estudos com aplicação de vários TEsc com EV para se esclarecer esse efeito, semelhante ao realizado com o TC $6^{2,15-17}$.

Na padronização do TC6, houve preocupação com a utilização do estímulo verbal ${ }^{2}$, motivado por estudos que mostraram que o estímulo verbal pode aumentar a distância percorrida em até 30 metros $^{3,4}$ e assegura máxima motivação e performance do indivíduo ${ }^{2,18,19}$. Os resultados encontrados nesse trabalho mostraram que o EV também é fundamental para o resultado do TEsc.

Em pneumopatas, o uso do estímulo verbal melhora o resultado do TC6 quando comparado com os valores previstos $^{20,21}$. Mesmo conhecendo os efeitos do estímulo verbal no TC6, após revisão sistemática, observou-se que somente quatro relataram seu uso ${ }^{22}$, mostrando que a padronização é fundamental para comparar resultados e para utilizá-lo na prática clínica.

O estímulo verbal também é importante durante a ergoespirometria ${ }^{23}$, e o consumo de oxigênio $\left(\mathrm{VO}_{2}\right)$ é maior nos testes realizados com estímulo verbal de sujeitos destreinados ${ }^{24}$ e quando realizado no início do teste ${ }^{25}$. Finalmente, testes anaeróbicos em bicicleta também sofrem influência do estímulo verbal ${ }^{26}$.

Pesquisas que envolvem o TEsc não seguem um padrão de investigação quanto à variável de controle. Os autores utilizam a quantidade de lances ${ }^{27}$, ou o tempo ${ }^{5-7}$, ou a altura ${ }^{27-31} \mathrm{e}, \mathrm{em}$ alguns casos, a velocidade no TEsc ${ }^{32}$. Para predizer o risco de complicação cirúrgica, a escolha pelo tempo no TEsc justifica-se, pois acreditamos, assim como outros autores ${ }^{5,6}$, que um sujeito que sobe a escada em 30 segundos tem melhor condição cardiorrespiratória se comparado a outro que demora 120 segundos para subir a mesma altura, por exemplo.
A falta de padronização em relação à variável a ser estudada, ao tamanho da escada, assim como ao tipo de EV utilizado, dificulta a comparação entre os estudos. Tanto para fins científicos como para que o TEsc passe a fazer parte da rotina de avaliação do paciente cirúrgico ou de outras doenças respiratórias, a padronização do TEsc é importante.

Para comparar nossos resultados com outros estudos que usaram o tempo no TEsc, deve-se utilizar a velocidade de subida já que em nosso estudo a altura da escada era inferior a 12 metros. Assim, os indivíduos aqui estudados demoraram 21 segundos para subir uma escada com 7,16 metros com estímulo verbal (velocidade $0,34 \mathrm{~m} / \mathrm{seg}$ ), resultados iguais a pacientes cirúrgicos que não complicaram no pós-operatório de toracotomia ${ }^{33}$, os quais demoraram 35 segundos para subir uma escada com 12,16 metros (velocidade 0,34 m/seg), mostrando que, mesmo em escadas menores, a exigência dos testes foi semelhante em relação à velocidade.

Diante do exposto, outros estudos podem ser propostos a fim de fixar a altura mínima para que a exigência cardiorrespiratória seja atingida usando a velocidade como variável de controle. Isso seria possível avaliando as velocidades atingidas em diferentes escadas e alturas, verificando se o indivíduo mantém essa velocidade e se as variáveis cardiorrespiratórias se alteram de forma similar diante das diversas situações.

Neste estudo, as variáveis cardiorrespiratórias (PAS, FR e P) e a sensação de dispneia (Escala de Borg) aumentaram significativamente nos pós-testes, sejam estes com ou sem EV. Observando os valores da frequência cardíaca atingida no final do teste em relação à máxima, pudemos observar que a sobrecarga chegou a 80\% da máxima, mostrando que a sobrecarga cardiorrespiratória exigida no TEsc é importante.

Considerando que o tempo de duração dos testes foi de 21 segundos em média, acreditamos que a via metabólica aqui utilizada é a via anaeróbica láctica e que o tempo de repouso foi suficiente para recuperação cardiorrespiratória e muscular ${ }^{34}$, porém outros trabalhos podem ser realizados utilizando, além das medidas dos sinais vitais, a medida de lactato sanguíneo.

Em trabalho semelhante, indivíduos com obstrução ao fluxo aéreo apresentaram FC, média da $\mathrm{PA}$ e $\mathrm{SpO}_{2}$ análogas a nossa amostra. A única variável respiratória com grande diferença foi a FR, a qual teve maior aumento, possivelmente decorrente do distúrbio obstrutivo da amostra ${ }^{35}$. A variação da Escala de Borg do TEsc com EV foi significativamente maior em relação à variação do TEsc sem EV, o que significa que a sensação 
de cansaço é maior quando com EV. Essa sensação é decorrente do maior esforço físico necessário para vencer a altura solicitada no teste em menor tempo.

Por ser o TEsc muito utilizado em pacientes cirúrgicos, testar os efeitos do EV seria importante também nessa população. Além disso, medidas diretas das vias metabólicas utilizadas durante os testes forneceriam informações adicionais a respeito de sua intensidade.

\section{CONCLUSÃO}

Adultos saudáveis melhoram o desempenho no teste de escada quando utilizado o estímulo verbal. As variáveis cardiorrespiratórias aqui estudadas comportaram-se de maneira fisiológica independentemente do estímulo. A sensação de esforço foi maior no teste realizado com estímulo verbal.

\section{REFERÊNCIAS}

1. Brunelli A, Refai M, Xiumé F, Salati M, Sciarra $V$, Socci L, et al. Performance at symptom-limited stair-climbing test is associated with increased cardiopulmonary complications, mortality, and costs after major lung resection. Ann Thorac Surg. 2008;86(1):240-8.

2. Brooks D, Solway S, Gibbons WJ. ATS statement: guidelines for the six-minute walk test. Am J Respir Crit Care Med. 2002;166(1):111-7.

3. Guyatt GH, Pugsley SO, Sullivan MJ, Thompson PJ, Berman L, Jones $\mathrm{NL}$, et al. Effect of encouragement on walking test performance. Thorax. 1984;39(11):818-22.

4. Guyatt GH, Sullivan MJ, Thompson PJ, Fallen EL, Pugsley SO, Taylor DW, et al. The 6-minute walk: a new measure of exercise capacity in patients with chronic heart failure. CMAJ. 1985; 132(8):919-23.

5. Cataneo DC, Kobayasi S, Carvalho LR de, Paccanaro RC, Cataneo AJM. Accuracy of six minute walk test, stair testand spirometry using maximal oxygen uptake as gold standard. Acta Cir Bras. 2010;25(2):194-200.

6. Cataneo DC, Cataneo AJM. Acurácia do teste de escada utilizando o consumo máximo de oxigênio como padrão-ouro. J Bras Pneumol. 2007:33(2):128-33.

7. Ambrozin ARP, Da Silva KJR. Influência do estímulo verbal no tempo do teste de escada. ASSOBRAFIR Ciência. 2011;2(1):19-28.

8. Win T, Groves AM, Ritchie AJ, Wells FC, Cafferty F, Laroche CM. The effect of lung resection on pulmonary function and exercise capacity in lung cancer patients. Respir Care. 2007;52(6):720-6.

9. Brunelli A, Refai M, Xiumé F, Salati M, Marasco R, Sciarra V, et al. Oxygen desaturation during maximal stair-climbing test and postoperative complications after major lung resections. Eur J Cardiothorac Surg. 2008;33(1):77-82.

10. Baecke JA, Burema J, Frijters JE. A short questionnaire for the measurement of habitual physical activity in epidemiological studies. Am J Clin Nutr. 1982;36(5):936-42.
11. World Health Organization. Physical status: the use and interpretation of anthropometry. In: Report of a WHO Expert Committee. WHO Technical Report Series 854. 1995. p. 36.

12. Standardization of Spirometry, 1994 Update. American Thoracic Society. Am J Respir Crit Care Med. 1995;152(3):1107-36.

13. Mion Jr. D, Gomes MAM, Nobre F, Amodeo C, Kohlmann Jr. O, Praxedes JN, et al. IV diretrizes brasileiras de hipertensão arterial. Arq Bras Cardiol 2004:82(Suppl 4):7-14.

14. Wilson RC, Jones PW. A comparison of the visual analogue scale and modified Borg scale for the measurement of dyspnoea during exercise. Clin Sci. 1989;76(3):277-82

15. Rodrigues SL, Mendes HFE, Viegas CAA. Teste de caminhada de seis minutos: estudo do efeito do aprendizado em portadores de doença pulmonar obstrutiva crônica. J Bras de Pneumol. 2004;30(2):121-5.

16. Knox AJ, Morrison JF, Muers MF. Reproducibility of walking test results in chronic obstructive airways disease. Thorax. 1988;43(5):388-92.

17. Elpern EH, Stevens D, Kesten S. Variability in performance of timed walk tests in pulmonary rehabilitation programs. Chest. 2000;118(1):98-105.

18. Troosters T, Gosselink R, Decramer M. Six minute walking distance in healthy elderly subjects. Eur Respir J. 1999;14(2):270-4.

19. Rodrigues SL, Viegas CAA. Estudo de correlação entre provas funcionais respiratórias e o teste de caminhada de seis minutos em pacientes portadores de doença pulmonar obstrutiva crônica. J Bras Pneumol. 2002;28(6):324-8.

20. Enright PL, Sherrill DL. Reference equations for the six-minute walk in healthy adults. Am J Respir Crit Care Med. 1998:158(5 Pt 1):1384-7.

21. Rodrigues SL, Mendes HF, Viegas CA. Teste de caminhada de seis minutos: estudo do efeito do aprendizado em portadores de doença pulmonar obstrutiva crônica. J Pneumol. 2004;30(2):121-5.

22. Graham JE, Ostir GV, Fisher SR, Ottenbacher KJ. Assessing walking speed in clinical research: a systematic review. J Eval Clin Pract. 2008;14(4):552-62.

23. Chitwood LF, Moffatt RJ, Burke K, Luchino P, Jordan JC. Encouragement during maximal exercise testing of type A and type B scorers. Percept Mot Skills. 1997:84(2):507-12

24. Moffatt RJ, Chitwood LF, Biggerstaff KD. The influence of verbal encouragement during assessment of maximal oxygen uptake. J Sports Med Phys Fitness. 1994;34(1):45-9.

25. Andreacci JL, LeMura LM, Cohen SL, Urbansky EA, Chelland SA, Von Duvillard SP. The effects of frequency of encouragement on performance during maximal exercise testing. J Sports Sci. 2002;20(4):345-52

26. Karaba-Jakovljević D, Popadić-Gaćesa J, Grujić N, Barak O, Drapsin M. Motivation and motoric tests in sports. Med Pregl. 2007;60(5-6):231-6

27. Girish M, Trayner E, Dammann O, Pinto-Plata V, Celli B. Symptomlimited stair climbing as a predictor of postoperative cardiopulmonary complications after high-risk surgery. Chest. 2001;120(4):1147-51.

28. Olsen G, Bolton J, Weiman D, Hornung C. Stair climbing as an exercise test to predict the postoperative complications of lung resection. Two years' experience. Chest. 1991;99(3):587-90.

29. Reilly JJ. Benefits of aggressive perioperative management in patients undergoing thoracotomy. Chest. 1995;107(6 Suppl):312S-5S. 
30. Brunelli A, Monteverde M, Salati M, Borri A, Al Refai M, Fianchini A Stair-climbing test to evaluate maximum aerobic capacity early after lung resection. Ann Thorac Surg. 2001;72(5):1705-10.

31. Holden D, Rice T, Stelmach K, Meeker D. Exercise testing, 6-min walk, and stair climb in the evaluation of patients at high risk for pulmonary resection. Chest. 1992:102(6):1774-9.

32. Koegelenberg CFN, Diacon AH, Irani S, Bolliger CT. Stair climbing in the functional assessment of lung resection candidates. Respiration. 2008;75(4):374-9.
33. Cataneo DC, Ambrozin ARP, Arruda K, Cateno AJM. Assessment of six-minute walk test and stair-climbing test time as predictors of postthoracotomy complications. Chest. 2010;138:569A.

34. American Thoracic Society/American College of Chest Physicians. ATS/ACCP statement on cardiopulmonary exercise testing. Am J Respir Crit Care Med. 2003:167:211-77.

35. Pollock M, Roa J, Benditt J, Celli B. Estimation of ventilatory reserve by stair climbing. A study in patients with chronic airflow obstruction. Chest. 1993;104(5):1378-83. 